\title{
Efecto del pH sobre propiedades mecánicas, fisicoquímicas y morfológicas en películas comestibles multicomponentes
}

\section{Effect of pH on mechanical, physicochemical and morphological properties in multicomponent edible films}

\author{
Jhusty Meliza Moreno Henao ${ }^{1}$; Yanneth Bohórquez Pérez ${ }^{2}$; Leidy Ayala Sánchez ${ }^{3}$; Claudia Valenzuela Real ${ }^{4}$
}

11.ng. Agroindustrial, c.M.Sc. Universidad del Tolima, Ibagué - Tolima, Colombia; e-mail: jmmorenoh@ut.edu.co; Dhttps://orcid.org/0000-0003-2861-2474

${ }^{2}$ Ing. Industrial M.Sc. Universidad del Tolima, Programa Ingeniería Agroindustrial y Maestría en Ciencias Agroalimentarias, Ibagué - Tolima, Colombia; e-mail: ybohorq@ut.edu.co; Dhttps://orcid.org/0000-0002-5348-6345

${ }^{3}$ Ing. Agroindustrial, M.Sc., Servicio Nacional de Aprendizaje - SENA, Facilitadora de Innovación Biotecnología y Nanotecnología, Ibagué - Tolima, Colombia; e-mail: carolinayala17@misena.edu.co; Dhttps://orcid.org/0000-0002-6839-8786

${ }^{4}$ Ing. Industrial, M.Sc. Universidad del Tolima, Programa Ingeniería Agroindustrial y Maestría en Ciencias Agroalimentarias, Ibagué - Tolima, Colombia; e-mail: cpvalenz@ut.edu.co; Dhttps://orcid.org/0000-0003-4750-0259

Cómo citar: Moreno Henao, J.M.; Bohórquez Pérez, Y.; Ayala Sánchez, L.; Valenzuela Real, C. 2020. Efecto del pH sobre propiedades mecánicas, fisicoquímicas y morfológicas en películas comestibles multicomponentes. Rev. U.D.C.A Act. \& Div. Cient. 23(1):e1457. http://doi.org/10.31910/rudca.v23.n1.2020.1457

Artículo de acceso abierto publicado por Revista U.D.C.A Actualidad \& Divulgación Científica, bajo una licencia Creative Commons CC BY-NC 4.0

Publicación oficial de la Universidad de Ciencias Aplicadas y Ambientales U.D.C.A, Institución de educación superior Acreditada de Alta Calidad por el Ministerio de Educación Nacional.

Recibido: Agosto 27 de 2018

Aceptado: Enero 22 de 2020

Editado por: Ingeborg Zenner de Polanía

\section{RESUMEN}

El pH es un parámetro fundamental a considerarse en el momento de diseñar películas multicomponentes, debido a que interviene en la compatibilidad e interacciones que se generan entre los componentes de la matriz, determina la funcionalidad de las mismas y como se demuestra en este estudio, el $\mathrm{pH}$ tiene una influencia significativa en las propiedades mecánicas, fisicoquímicas y morfologías de las películas. Con ese objetivo, se sometieron películas a base de goma gellan, gelatina, caseinato de calcio, aceite de canola, glicerol, tween 80 y natamisina, a cuatro niveles de pH T1:6,6 T2:6,2 T3:5,8 T4:5,4 para evaluar y determinar la influencia del $\mathrm{pH}$ en las propiedades finales de las películas. Como resultado obtenido en las propiedades, se presentaron diferencias significativas $(\alpha=0,05)$, influenciadas por la modificación del $\mathrm{pH}$, de modo que, en las películas sometidas a un $\mathrm{pH}$ de $\mathrm{T} 4=5,4$ se generó una mejor compatibilidad de los componentes, por las condiciones del medio que permitieron obtener interacciones por fuerzas electrostáticas entre los polímeros y el aceite, evidenciándose en el mejor comportamiento mecánico con una elongación de $10,6 \pm 1,8 \%$, mayor permeabilidad al vapor de agua, solubilidad de $51 \pm 0,02 \%$ y un contenido de humedad de $9 \pm 3,0 \%$; a diferencia de las películas de T1, en las cuales, predominaron las interacciones a través de los enlaces de hidrógeno, afectando la permeabilidad y el comportamiento mecánico de las mismas. En películas multicomponentes a base polímeros y lípidos para garantizar mayor compatibilidad e interacciones que se reflejan en mejores propiedades mecánicas, fisicoquímicas y morfológicas entre los componentes se deben elaborar películas a $\mathrm{pH}$ de 5,4 o, en 
caso contrario, en los cuales, las propiedades deban permitir mayores interacciones entre grupos polares de los componentes utilizar $\mathrm{pH}$ más básicos, como pH de 6,6.

Palabras clave: biopolímeros; compatibilidad; interacciones; permeabilidad; elasticidad.

\section{ABSTRACT}

$\mathrm{pH}$ is a fundamental parameter to be considered when designing multicomponent films, because it intervenes in the compatibility and interactions that are generated between the components of the matrix, determines their functionality and as demonstrated in this study the $\mathrm{pH}$ has a significant influence on the mechanical, physicochemical and morphological properties of films. With this objective, films based on gellan gum, gelatin, calcium caseinate and canola oil were subjected to four levels of $\mathrm{pH}$ T1 $=6.6$ T2 $=$ 6.2 T3 $=5.8 \mathrm{~T} 4=5.4$ to evaluate and determine the influence of $\mathrm{pH}$ on the final properties of the films. As a result obtained in the properties there were significant differences $(\alpha=0.05)$ influenced by the modification of the $\mathrm{pH}$ so that in the films subjected to a $\mathrm{pH}$ of $\mathrm{T} 4=5.4$ a better compatibility of the components was generated by the conditions of the medium that allowed to obtain interactions by electrostatic forces between the polymers and the oil, being evident in the best mechanical behavior with an elongation of $10.6 \pm 1.8 \%$, greater water vapor permeability, solubility of 51 $\pm 0.02 \%$ and a moisture content of $9 \pm 3.0 \%$; unlike the T1 films in which the interactions through hydrogen bonds predominated, affecting their permeability and mechanical behavior. Finally, as a conclusion in multi-component films based on polymers and lipids to ensure greater compatibility and interactions that are reflected in better mechanical, physicochemical and morphological properties between the components, films at $\mathrm{pH} 5.4$ or otherwise in the which properties should allow greater interactions between polar groups of the components to use more basic $\mathrm{pH}$ such as $\mathrm{pH}$ 6.6.

Keywords: biopolymers; compatibility; interactions; permeability; elasticity.

\section{INTRODUCCIÓN}

Se estima que las pérdidas poscosecha de los productos hortofrutícolas que se producen en el mundo son debido a deterioros microbiológicos y fisiológicos, que se producen como consecuencia de factores de orden tecnológico en la cadena de distribución. Ante esta situación y con el objetivo de disminuir el efecto de los factores citados y prolongar la vida poscosecha de los productos hortofrutícolas, se han investigado tecnologías relacionadas con la conservación de alimentos que involucran la formulación y la elaboración de recubrimientos y películas comestibles, para mejorar la calidad y valor comercial de los productos, como barrera a la humedad y a gases, resistencia mecánica, vehículo en la liberación de diversos aditivos y compuestos activos, como agentes antimicrobianos, antifúngicos y antioxidantes, facilidad en su aplicación, bajos costos de producción y gran variedad de materiales existentes para su preparación (Yildirim et al. 2019; Khodaei et al. 2019).
Los biopolímeros son los principales materiales de constitución que se han utilizado para esta tecnología alternativa en la conservación de alimentos y, con el fin de mejorar la funcionalidad de estos materiales, se han enfocado sus esfuerzos en el diseño de películas que combinen más de un componente estructural y así lograr efectos sinérgicos entre los mismos (Hassan et al. 2018). Para esto, diferentes estudios evalúan la compatibilidad de los componentes, teniendo en cuenta su estructura química, cargas eléctricas, grado de interacción, orden de mezcla y los parámetros, que permiten una adecuada interacción entre los componentes, tales como $\mathrm{pH}$ y fuerza iónica (Manrich et al. 2017).

Los hidrocoloides (proteína - polisacárido) son biopolímeros utilizados como matriz estructural en las películas por ser una excelente barrera al oxígeno y aromas, pero no se comportan bien como barreras de humedad, debido a su naturaleza hidrofílica, que se atribuye a la ordenada estructura en la red de enlaces de hidrógeno. Por tal razón, una forma de mejorar las propiedades de resistencia al agua es la adición de compuestos hidrofóbicos, tales como los lípidos, excelentes barreras contra la migración de humedad y combinados con hidrocoloides pueden tener además una mayor permeabilidad a la humedad en comparación con la de los lípidos puros (Bonilla et al. 2012; Cazón et al. 2017; Jiménez et al. 2012)

$\mathrm{El} \mathrm{pH}$ es un factor determinante para las interacciones que se generan entre los componentes de las películas y han sido investigadas, principalmente, en los complejos proteínas - polisacáridos. McClements (2005) indicó que las interacciones entre proteínas y polisacáridos en un entorno acuoso pueden ser repulsivas o atractivas y dependen principalmente del $\mathrm{pH}$, que modifica las cargas eléctricas en los biopolímeros. En un estudio realizado por De Kruif et al. (2004), se evidenció que, cuando el pH estuvo cercano al punto isoeléctrico de las proteínas (PI), las moléculas se atraen y permiten la formación de complejos solubles electrostáticos proteína-polisacárido, a diferencia de cuando se alejan del PI, que se generan interacciones mediante enlaces del grupo $\mathrm{OH}^{-}$.

Estas interacciones entre las proteínas - polisacáridos son fundamentales para la formación de las películas; sin embargo, mejorar las propiedades y las características finales de las películas multicomponentes, a través de condiciones adecuadas de $\mathrm{pH}$ para lograr interacciones atractivas entre los componentes, ha sido relevante en pocas investigaciones y, en la mayoría de estudios, la verificación de la compatibilidad de los materiales y las diferentes interacciones que se presenten entre ellos, incluyendo la adición de lípidos a los complejos poliméricos, se ha limitado a los resultados en las propiedades y características finales de las películas (Pan et al. 2014).

Esteghlal et al. (2018) evaluaron las interacciones electrostáticas entre gelatina y carboximetilcelulosa (CMC) en función del $\mathrm{pH}$ y la relación de la mezcla de los componentes, para observar cómo estas interacciones afectan las propiedades físicas y mecánicas de las películas compuestas. Pereda et al. (2011), en películas compuestas de gelatina con quitosano, demostraron que, en condiciones adecuadas de $\mathrm{pH}$, se generan interacciones atractivas electrostáticas entre los dominios aniónicos/catiónicos de los componentes 
que se evidencian en las mejoras de las propiedades mecánicas y fisicoquímicas. También, Cao et al. (2007), en películas de gelatina - aislado de proteína de soya, encontraron que las condiciones del $\mathrm{pH}$ en la solución afecta directamente la formación de las películas y sus propiedades finales.

Por lo tanto, someter películas multicomponentes a diferentes condiciones de $\mathrm{pH}$ para optimizar sus propiedades finales vislumbra la importancia de este estudio, en el cual, se evaluó la influencia del $\mathrm{pH}$ en propiedades mecánicas, fisicoquímicas y morfológicas de películas multicomponentes.

\section{MATERIALES Y MÉTODOS}

Preparación de películas comestibles multicomponente. Se obtuvo películas para caracterización, mediante el método de casting o preformado. El $\mathrm{pH}$ del agua destilada se ajustó en cuatro tratamientos de la siguiente forma T1: 6,6 - T2: 6,2 - T3: 5,8 - T4: 5,4 mediante la adición de soluciones de citrato de sodio $(40 \%)$ y ácido cítrico (10\%) y una vez ajustado el $\mathrm{pH}$ del agua destilada, se solubilizó de manera independiente cada polímero, de acuerdo con las concentraciones y las condiciones adecuadas para cada uno, durante 5 minutos en agitación magnética: goma gellan $\left(0,5 \% ; 90^{\circ} \mathrm{C}\right.$; $5000 \mathrm{rpm})$, gelatina $\left(0,25 \% ; 50^{\circ} \mathrm{C} ; 2000 \mathrm{rpm}\right)$ y caseinato de calcio $\left(0,10 \% ; 30^{\circ} \mathrm{C} ; 1000 \mathrm{rpm}\right)$ (Arrieta et al. 2013; Chambi \& Grosso, 2006; Lee et al. 2004). Luego de solubilizados los componentes fueron mezclados, de acuerdo con el siguiente orden: se incorporó a la gelatina, caseinato de calcio, goma gellan, seguido del glicerol $(0,1 \%)$, tween $80(0,25 \%)$, aceite de canola $(0,025 \%)$ y natamisina (100 ppm), para obtener la mezcla, mediante homogenización empleando un Ultraturrax (T25, IKA, Alemania), a 7000rpm, a la cual, se le midió el $\mathrm{pH}$ y se vertieron en placas poliacrílicas con volúmenes constantes de $250 \mathrm{ml}$ de solución por $\mathrm{cm}^{2}$ para moldear. La obtención de las películas, se realizó por secado en condiciones de refrigeración $\left(3 \pm 2^{\circ} \mathrm{C}\right.$, HR: $75 \pm 5 \%$ ), durante $72 \mathrm{~h}$, cumplido el tiempo las películas obtenidas, se retiraron de las placas y se acondicionaron en un desecador vertical, con $\mathrm{Mg}\left(\mathrm{NO}_{3}\right)_{2} \cdot 6 \mathrm{H}_{2} \mathrm{O}$ (HR: $50 \pm 3 \%, 24 \pm 2^{\circ} \mathrm{C}$ por $24 \mathrm{~h}$ ), previo a su caracterización.

\section{Caracterización de las películas preformadas:}

Espesor. Se calculó como la media aritmética de 10 medidas, tomadas en diferentes puntos de tiras de película, utilizando un micrómetro Mitutoyo (Japón), de 0,01 mm de precisión (Galus \& Lenart, 2013).

Propiedades mecánicas. La resistencia a la extensión (RT) (N), tensión al rompimiento (TRo) (MPa) y elongación a la fractura (EF) (\%), se determinaron usando un Texturometro LS1 (LLOYD, UK) y el software NexygenPlus. Las muestras, se cortaron en segmentos rectangulares de $80 \times 25 \mathrm{~mm}$ y se analizaron siguiendo la metodología ASTM International (2002), con una celda de carga de $20 \mathrm{~N}$, mordazas de $25 \times 25 \mathrm{~mm}, 50 \mathrm{~mm}$ de separación inicial de agarre y velocidad de $50 \mathrm{~mm} / \mathrm{min}$, con tres réplicas por tratamiento y utilizando el espesor medio de cada película.
Permeabilidad al vapor de agua (PVA). Se determinó gravimétricamente utilizando el método E-96-80, con las modificaciones efectuadas por Gennadios et al. (1994), para películas hidrofilicas, empleando celdas de permeabilidad. Las muestras de película, se ubicaron en la parte superior de las en celdas que contenían $6 \mathrm{~mL}$ de agua destilada (HR: $100 \%$ ), se sellaron herméticamente, aplicando parafina fundida y se llevaron a un desecador con $\mathrm{NaCl}$ (HR: $75 \pm 3 \%$ ). Se registró el peso cada hora durante ocho horas. La PVA (g/m s Pa) fue calculada según la ecuación 1.

$\mathrm{PVA}=\mathrm{aEs} / \mathrm{tAP}_{\mathrm{sat}}\left(\mathrm{HR}_{1}-\mathrm{HR}_{2}\right)$

ecuación 1

Donde, a es la masa de la celda de permeabilidad (g) al tiempo $\mathrm{t}(\mathrm{s})$; Es representa el espesor de la muestra (m); A es el área de exposición $\left(\mathrm{m}^{2}\right)$, Psat es la presión de vapor saturado a la temperatura del análisis $(\mathrm{Pa}) ; \mathrm{HR}_{1}$ es la humedad relativa del agua contenida en la celda, expresada como fracción y $\mathrm{HR}_{2}$ es la humedad relativa de la cámara, expresada como fracción.

Contenido de humedad (CH) y solubilidad (SA). El contenido de humedad $(\mathrm{CH})$ de las películas, se determinó mediante la metodología reportada por Ahmadi et al. (2012). Muestras de 20x20mm fueron pesadas (mi) y desecadas a $103 \pm 2^{\circ} \mathrm{C}$, durante $24 \mathrm{~h}$ (mf). Para determinar la SA, las muestras secas (msi) fueron depositadas en frascos de vidrio, que contenían $30 \mathrm{~mL}$ de agua destilada y llevadas a agitación de 100rpm, durante $1 \mathrm{~h}$. Luego, la parte insolubilizada se pasó por un filtro cualitativo y se llevó a secado a $60^{\circ} \mathrm{C}$, durante $2 \mathrm{~h}$ (msf). El contenido de humedad y la solubilidad de las películas fue determinado usando las ecuaciones 2 y 3 respectivamente.

$\mathrm{CH}=\frac{\mathrm{mi}-\mathrm{mf}}{\mathrm{mi}} * 100$

ecuación 2

Donde, mi es la masa inicial de las películas y $m f$ es la masa seca de las películas.

$\mathrm{SA}=\frac{\mathrm{msi}-\mathrm{msf}}{\mathrm{msi}} * 100$

ecuación 3

Donde, msi es la masa seca inicial y msf es la masa insoluble seca final.

Transparencia (TR). Se realizó de acuerdo con la técnica descrita por Han \& Floros (1997), en tiras de película de 10x40mm, utilizando un espectrofotómetro UV-Vis, Genesys $10 \mathrm{~S}$ (Thermo Scientific ${ }^{\mathrm{TM}}$, EE.UU). La transparencia de las películas, se determinó en la longitud de $600 \mathrm{~nm}$ y de acuerdo con la ecuación 4.

$\mathrm{TR}=-\log \frac{\mathrm{T}_{600}}{\mathrm{x}}$

ecuación 4

Donde, T600 es la transmitancia a $600 \mathrm{~nm}$ y $x$ es el espesor de la película $(\mathrm{mm})$.

Microscopia de Fuerza Atómica (AFM). La morfología de la superficie de las películas, se analizó utilizando microscopia de fuerza atómica (AFM), a través de un microscopio de fuerza atómica Nanosurf (FlexAFM C3000), con un área de escaneo de 103x103 $\mu \mathrm{m}$. Se obtuvieron valores de rugosidad $\mathrm{Ra}(\mathrm{nm})$ y $\mathrm{Rq}(\mathrm{nm})$ 
(Ra: promedio del valor absoluto de las desviaciones de altura de una superficie media; Rq: media cuadrática promedio de las desviaciones de altura tomada de los datos de la media).

Análisis estadístico. El diseño experimental utilizado fue cuatro tratamientos y tres réplicas por tratamiento. Los resultados, se analizaron usando el paquete estadístico SPSS Statistics 21 para el software de Windows (SPSS Inc., Chicago, IL, EE. UU). Las diferencias estadísticamente significativas entre los valores medios de los resultados, se establecieron mediante un análisis de varianza (ANOVA), con un nivel de confianza del $95.0 \%(\alpha=0,05)$ y prueba múltiple de rangos con el estadígrafo Tukey.

\section{RESULTADOS Y DISCUSIÓN}

El pH y las propiedades mecánicas de películas multicomponentes. La ocurrencia de los fenómenos que se presentaron en las películas, se puede explicar porque el $\mathrm{pH}$ afectó la naturaleza de los materiales, la compatibilidad y las interacciones y relaciones que se presentaron en la matriz, incidiendo en el comportamiento mecánico de las películas, como se presentan en la tabla 1. La resistencia a la tracción (TR) y la tensión al rompimiento (TRo), que indica la resistencia mecánica, se presentó en el siguiente orden: T4 $<$ T3 $<$ T $2<\mathrm{T} 1$, a diferencia del $\%$ de elongación $(\% \mathrm{Ef})$, que fue $\mathrm{T} 1<\mathrm{T} 2<\mathrm{T} 3<\mathrm{T} 4$, demostrando que la compatibilidad entre los componentes afecta la resistencia o elasticidad la película, es decir, hace que red sea más elástica o más resistente (Esteghlal et al. 2018).

Chambi \& Grosso (2006), en películas de gelatina - caseinato, encontraron que la elasticidad de las películas (\%Ef) se asocian, en gran medida, con la compatibilidad de los componentes y el $\mathrm{pH}$, que afecta la distribución de los grupos laterales para generar más interacciones entre las proteínas y con el plastificante, por esta razón, en los tratamientos T3 y T4, por las interacciones electrostáticas de los dominios aniónicos del polisacárido y catiónicos de las proteínas, se liberaron en el polisacárido los grupo $\mathrm{OH}^{-}$, que permitieron mayor interacción con el plastificante y los lípidos, mediante el tensoactivo, lo cual, se evidenció en la elasticidad y la flexibilidad de las películas (\%EF) (Qi et al. 2016), contrario a lo que ocurrió a medida que el pH aumentó, donde se observaron películas más resistentes y menos elásticas, como se evidenció en las películas del T1; en estos tratamientos, los materiales formaron una red más estable y organizada por las interacciones predominantes del grupo $\mathrm{OH}^{-} \mathrm{o}$ enlaces hidrógeno entre proteínas - polisacáridos, que limitaron la acción e interacción con el plastificante y el lípido, dando como resultado películas más resistentes.

Resultados similares reportaron Cao et al. (2007), quienes al estudiar el efecto del $\mathrm{pH}$ en películas de gelatina - proteína de soya encontraron una influencia significativa del pH en la conformación estructural de la película, que modificó sus propiedades mecánicas; las cifras revelaron que \%EF aumentó mientras TR y TRo disminuyó en pH más ácidos o cercanos al PI de las proteínas. Tongnuanchan et al. (2013), en películas de gelatina con aceites esenciales, también reportaron que la interacción de los lípidos entre las cadenas de péptidos reduce la alineación ordenada de la red, mejorando la flexibilidad y la elasticidad de las películas.

El pH y las propiedades fisicoquímicas de las películas multicomponentes. Las propiedades fisicoquímicas de las películas multicomponentes, que tienen como base estructural hidrocoloides, están altamente influenciadas por la naturaleza hidrofílica de los mismos; sin embargo, modificar el pH, el orden de adicción de los componentes y la presencia de un lípido permite mejorar las propiedades fisicoquímicas de los materiales con diferencias significativas, como se observa en la tabla 2.

Permeabilidad de películas multicomponentes. La estructura polimérica de las películas basadas en hidrocoloides, por su naturaleza hidrofílica son, generalmente, pobres barreras a la humedad y a la transmisión del vapor de agua (PVA). La modificación del pH intervino en la formación de las redes de la matriz polimérica por el ordenamiento de los componentes, que incidió en la migración de agua en las películas (Garavand et al. 2017). En los tratamientos T4 y T3, se presentaron varios fenómenos, que disminuyeron la transferencia de vapor de agua; primero, la formación de un complejo proteico entre el caseinato de calcio y la gelatina, que permitió la interacción de los iones divalentes de $\mathrm{Ca}^{+2}$ con el polisacárido, para formar una red proteína - polisacárido y, segundo, la interacción del polisacárido con el tensoactivo y el aceite, lo cual, limitó la movilidad de los componentes en la matriz y como resultado, se obtuvo la mejor barrera al vapor de agua (Arrieta et

Tabla 1. Comportamiento mecánico de las películas comestibles multicomponentes.

\begin{tabular}{|c|c|c|c|c|c|}
\hline Tratamiento & $\mathbf{p H}$ & Espesor (mm) & $\begin{array}{c}\text { TR. Resistencia } \\
\text { a la extensión (N) }\end{array}$ & $\begin{array}{c}\text { TRo. Tensión al } \\
\text { rompimiento (MPa) }\end{array}$ & $\begin{array}{c}\text { \%Ef. Elongación } \\
\text { a la fractura }\end{array}$ \\
\hline T1 & 6,6 & $0,045 \pm 0,005 \mathrm{a}$ & $15,6 \pm 1,4 \mathrm{a}$ & $14,5 \pm 3,2 \mathrm{a}$ & $6,6 \pm 2,8 \mathrm{a}$ \\
\hline $\mathrm{T} 2$ & 6,3 & $0,043 \pm 0,006 \mathrm{a}$ & $11,0 \pm 2,5 \mathrm{a}$ & $13,7 \pm 2,0 \mathrm{a}$ & $7,1 \pm 2,0 \mathrm{a}$ \\
\hline $\mathrm{T} 3$ & 5,8 & $0,041 \pm 0,003 \mathrm{a}$ & $8,9 \pm 1,6 \mathrm{~b}$ & $11,0 \pm 2,6 \mathrm{ab}$ & $7,7 \pm 1,8 \mathrm{a}$ \\
\hline $\mathrm{T} 4$ & 5,4 & $0,036 \pm 0,004 \mathrm{~b}$ & $6,3 \pm 1,2 \mathrm{~b}$ & $7,3 \pm 1,4 \mathrm{~b}$ & $10,6 \pm 1,8 \mathrm{~b}$ \\
\hline
\end{tabular}

Letras diferentes en la misma columna indican diferencias significativas $(\alpha=0,05)$. Los resultados son medias con su respectiva desviación estándar. 
Tabla 2. Propiedades fisicoquímicas de las películas comestibles multicomponentes.

\begin{tabular}{|c|c|c|c|}
\hline Tratamientos & $\begin{array}{c}\text { Permeabilidad } \\
\text { (gmm/m2diakPa) }\end{array}$ & $\begin{array}{c}\text { Contenido de } \\
\text { humedad (\%) }\end{array}$ & Solubilidad (\%) \\
\hline T1 & $3,7 \pm 0,4 \mathrm{a}$ & $16 \pm 2,0 \mathrm{a}$ & $76 \pm 2,0 \mathrm{a}$ \\
\hline T2 & $3,3 \pm 0,6 \mathrm{ab}$ & $13 \pm 0,5 \mathrm{ab}$ & $69 \pm 1,5 \mathrm{~b}$ \\
\hline T3 & $2,4 \pm 0,4 \mathrm{bc}$ & $10 \pm 1,0 \mathrm{bc}$ & $58 \pm 1,0 \mathrm{c}$ \\
\hline T4 & $1,8 \pm 0,2 \mathrm{c}$ & $9 \pm 3,0 \mathrm{c}$ & $51 \pm 4,0 \mathrm{c}$ \\
\hline
\end{tabular}

Letras diferentes en la misma columna indican diferencias significativas $(\boldsymbol{\alpha}=0,05)$. Los resultados son medias con su respectiva desviación estándar.

al. 2014), a diferencia de los tratamientos T1 y T2, que presentaron diferencias significativas $(\mathrm{p}>0,05)$ con $\mathrm{T} 4 \mathrm{y}$ fueron barreras menos eficientes a la humedad; en estos tratamientos, las interacciones predominantes entre los componentes disminuyeron la relación con el aceite y permitieron mayor relación de los polímeros entre los enlaces $\mathrm{OH}^{-}$y las moléculas de agua (Cerqueira et al. 2012).

Contenido de humedad y solubilidad de las películas comestibles. El contenido de humedad es una propiedad relacionada con el volumen ocupado por las moléculas de agua en la red estructural de la película multicomponente; no obstante, el $\mathrm{pH}$ influye en la capacidad de retención de agua de la red estructural en las películas. En T4, la creación del complejo polimérico y la interacción con el lípido otorgan menores capacidades en la atracción y retención de agua en la red estructural, a diferencia de los tratamientos de T1 y T2 que, por las interacciones a través de los enlaces de hidrógeno, existe mayor afinidad por las moléculas de agua, lo que permite retenerlas fácilmente dentro de la matriz (Sanyang et al. 2016).

Del mismo modo, los resultados obtenidos, se compararon con otros estudios de películas de naturaleza hidrofílica, que también correlacionan el contenido de humedad con la formación de interacciones polares y no polares en la matriz estructural. En Ghasemlou et al. (2011), se reportó un aumento significativo del contenido de humedad de $23,59 \%$ a 37,04\% al aumentar las interacciones, a través de enlaces $\mathrm{OH}^{-}$y puentes de hidrógeno en películas preparadas a partir de kefiran con glicerol, mientras en Acosta et al. (2015)barrier and mechanical, la humedad de las películas de almidón - gelatina con la adición de aceites esenciales de canela, como componente hidrofóbico, el contenido de humedad disminuyó, de 11 a 7,7\%.

Por otro lado, la solubilidad, analizada por diferentes autores como una medida de resistencia al agua e integridad de una película, ésta determinada por la composición química de la matriz estructural y la naturaleza de sus componentes (Ahmadi et al. 2012). Los resultados incluidos en la tabla 2 , evidencian que las películas del T1 presentan una alta solubilidad, con un $76 \%$ de porcentaje de película solubilizado en agua; a diferencia de las películas del T4, en el cual, el porcentaje de solubilidad fue solo del 51\%. Resultados similares, se encontraron en películas de gellan - gelatina, referenciadas por Lee et al. (2004), quienes reportaron un porcentaje de solubilidad de 30 al 52\%, indicando la importancia de la formación de interacciones atractivas entre proteínas - polisacáridos - lípidos para formar soluciones menos polares. Las propiedades fisicoquímicas como solubilidad y contenido de humedad, son importantes para determinar la finalidad y los usos de las películas en productos alimenticios. Las películas poco solubles y con poca humedad son materiales apropiados, cuando es importante disminuir la pérdida de agua de los productos, como es el caso de las frutas y las hortalizas, mientras que las demás películas son adecuadas para tipos de envases que se deben disolver en agua antes de su uso (Jridi et al. 2014).

El pH y las propiedades ópticas de películas multicomponentes. Las propiedades ópticas de las películas son parámetros relevantes para determinar la aplicabilidad de las mismas, debido a su posible impacto en la apariencia de los productos a utilizar. La transparencia de las películas depende de la microestructura interna de la matriz, la distribución y la compatibilidad de los componentes, especialmente, de los agregados lipídicos. Algunos autores expresan que si las películas se comportan como un material homogéneo, la transmitancia de la luz es alta y las películas más transparentes; pero si las películas son heterogéneas, las transmitancia es baja y las películas menos transparentes (Rhim \& Shellhammer, 2005).

Las películas en estudio, en general, fueron materiales transparentes, presentando diferencias significativas $(\mathrm{p}>0,05)$ entre los tratamientos, como se detalla en la tabla 3. De los resultados, se observa que las películas más transparentes fueron las del T1, verificando que son películas con una red organizada en la matriz estructural, por las interacciones entre la red proteína - polisacárido - lípido, que genera películas más homogéneas y, por tanto, más permeables y solubles. A diferencia de las películas del T4, las cuales, fueron más opacas, posiblemente, por la formación de complejos de polielectrolitos (polianiones/cationes) y la distribución de los lípidos que generan películas más heterogéneas y, así, menos permeables y menos solubles (Monedero et al. 2010). 
Tabla 3. Propiedades ópticas de las películas comestibles multicomponentes

\begin{tabular}{|c|c|c|}
\hline Tratamientos & Transmitancia (\%) a $\mathbf{6 0 0} \mathbf{n m}$ & Transparencia \\
\hline T1 & $86,0 \pm 3,30 \mathrm{a}$ & $1,88 \pm 0,34 \mathrm{a}$ \\
\hline T2 & $62,2 \pm 9,92 \mathrm{ab}$ & $2,84 \pm 0,67 \mathrm{ab}$ \\
\hline T3 & $61,1 \pm 6,12 \mathrm{ab}$ & $3,13 \pm 0,29 \mathrm{ab}$ \\
\hline T4 & $51,8 \pm 1,78 \mathrm{~b}$ & $3,68 \pm 0,46 \mathrm{~b}$ \\
\hline
\end{tabular}

Letras diferentes en la misma columna indican diferencias significativas $(\alpha=0,05)$. Los resultados son medias con su respectiva desviación estándar.

En películas de gelatina - aislado proteína de soya, Cao et al. (2007) expresan que las películas preparadas a $\mathrm{pH} 8,10$, y 12, donde todos los compuestos eran solubles fueron más transparentes, a diferencia de las películas preparadas a $\mathrm{pH}$ de 6 , en que la proteína de soya era insoluble y las películas fueron más opacas. En películas de gelatina con aceites esenciales, Tongnuanchan et al. (2013) concluyeron que la disminución en la transmitancia de la luz fue causada, posiblemente, por la dispersión de luz de gotitas lipídicas, distribuidas por toda la red de proteínas.

El pH y la influencia en la morfología de películas multicomponentes. Gráficos en 3D, con área de escaneo $103 \times 103 \mu \mathrm{m}$, que se evidencian en la figura 1 y muestran la rugosidad de las superficies de las películas multicomponentes, se puede observar que las películas multicomponentes son heterogéneas, dada la falta de integración de la fase lipídica y la rápida cristalización, tanto de la natamisina como de los biopolímeros (Bierhalz et al. 2014)differential scanning calorimetry (DSC. Del mismo modo, se puede inferir que la rugosidad se asocia con la polaridad de las películas, la heterogeneidad y la transparencia de las películas, los cuales, se rigen por la formación de complejos y de redes, a través de la compatibilidad y las interacciones entre los componentes. Aquellas películas que se desarrollaron en el tratamiento T4 presentaron en un $62 \%$ mayor rugosidad que las películas del T1, lo que comprueba que la heterogeneidad del material se asocia con su alta barrera al vapor de agua, poca solubilidad y menor transparencia.

También, se observaron durante los análisis del AFM, presencia de poros en la mayoría de los gráficos observados. Este fenómeno, se pudo presentar, debido a la liberación de $\mathrm{CO}_{2}$ o liberación de calcio del caseinato en la matriz polimérica (Benavides et al. 2012). Esta presencia de poros puede resultar beneficiosa si las películas comestibles se van a utilizar en productos, como frutas, hortalizas o mínimamente procesados, para evitar la condensación o sobremaduración del producto.

Figura 1. Micrografías de AFM en 3D para las películas comestibles multicomponentes, en dimensiones de escaneo de $103 \mu \mathrm{m} x 103 \mu \mathrm{m}$.

T4

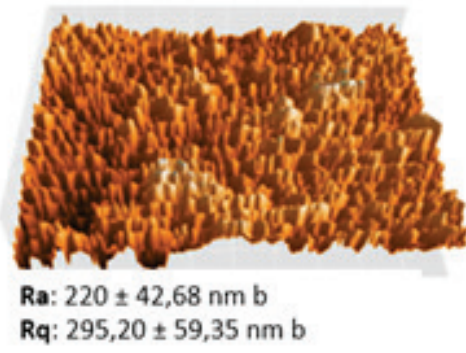

T2

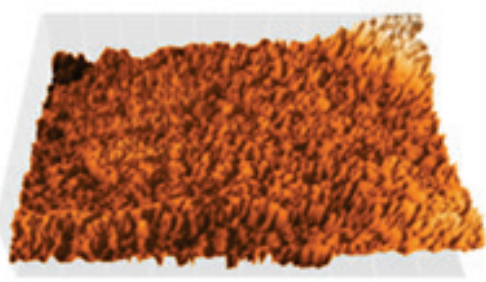

Ra: $123,65 \pm 10,97 \mathrm{~nm}$ a

Rq: $151,50 \pm 14,34 \mathrm{~nm}$ a
T3

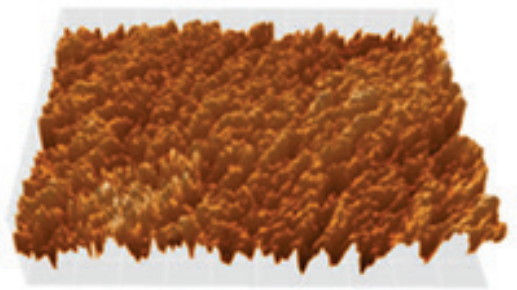

Ra: $84,76 \pm 6,42 \mathrm{~nm}$ a

Rq: $108,50 \pm 9,08 \mathrm{~nm}$ a

T4

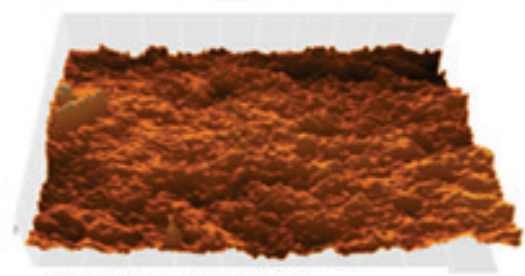

Ra: $70,96 \pm 17,72 \mathrm{~nm}$ a

$\mathrm{Rq}: 91,84 \pm 25,14 \mathrm{~nm}$ a 
De la presente investigación, se puede concluir que el pH es una variable que modifica las propiedades de las películas, debido a su incidencia en la compatibilidad y las interacciones entre los componentes, que conforman una matriz multicomponente; formular películas con $\mathrm{pH}$ adecuados aumentan las posibilidades de uso y de aplicación en la conservación de productos frescos y mínimamente procesados.

Agradecimientos. Las autoras agradecen al Laboratorio de Poscosecha de la Universidad del Tolima, por el apoyo en la elaboración de las pruebas y al Tecnoparque Nodo La Granja, Línea de Biotecnología y Nanotecnología, por la asesoría en las pruebas de microscopia de fuerza atómica (AFM). Conflicto de intereses: El manuscrito fue preparado y revisado con la participación de los autores, quienes declaramos que no existe conflicto de intereses que ponga en riesgo la validez de los resultados presentados. Financiación: El estudio fue financiado por el Comité Central de Investigaciones de la Universidad del Tolima.

\section{REFERENCIAS}

1. ACOSTA, S.; JIMÉNEZ, A.; CHÁFER, M.; GONZÁLEZMARTÍNEZ, C.; CHIRALT, A. 2015. Physical properties and stability of starch-gelatin based films as affected by the addition of esters of fatty acids. Food Hydrocolloids. (Netherlands). 49:135-143. https://doi.org/10.1016/j. foodhyd.2015.03.015

2. AHMADI, R.; KALBASI-ASHTARI, A.; OROMIEHIE, A.; YARMAND, M.-S.; JAHANDIDEH, F. 2012. Development and characterization of a novel biodegradable edible film obtained from psyllium seed (Plantago ovata Forsk). J. Food Engineering. (Netherlands). 109(4):745-751. https://doi. org/10.1016/j.jfoodeng.2011.11.010

3. ARRIETA, M.P.; PELTZER, M.A.; GARRIGÓS, M. DEL C.; JIMÉNEZ, A. 2013. Structure and mechanical properties of sodium and calcium caseinate edible active films with carvacrol. J. Food Engineering. 114(4):486-494. https:// doi.org/10.1016/j.jfoodeng.2012.09.002

4. ARRIETA, M.P.; PELTZER, M.A.; LÓPEZ, J.; GARRIGÓS, M. DEL C.; VALENTE, A.J.M.; JIMÉNEZ, A. 2014. Functional properties of sodium and calcium caseinate antimicrobial active films containing carvacrol. J. Food Engineering. 121:94-101. https://doi.org/10.1016/j. jfoodeng.2013.08.015

5. ASTM INTERNATIONAL. 2002. ASTM D882 - 02. Standard Test Method for Tensile Properties of Thin Plastic Sheeting.

6. BENAVIDES, S.; VILLALOBOS-CARVAJAL, R.; REYES, J.E. 2012. Physical, mechanical and antibacterial properties of alginate film: Effect of the crosslinking degree and oregano essential oil concentration. J. Food Engineering. 110(2):232239. https://doi.org/10.1016/j.jfoodeng.2011.05.023
7. BIERHALZ, A.C.K.; DA SILVA, M.A.; BRAGA, M.E.M.; SOUSA, H.J.C.; KIECKBUSCH, T.G. 2014. Effect of calcium and/or barium crosslinking on the physical and antimicrobial properties of natamycin-loaded alginate films. LWT - Food Science and Technology. (Estados Unidos). 57(2):494-501. https://doi.org/10.1016/j.lwt.2014.02.021

8. BONILLA, J.; ATARÉS, L.; VARGAS, M.; CHIRALT, A. 2012. Edible films and coatings to prevent the detrimental effect of oxygen on food quality: Possibilities and limitations. J. Food Engineering. 110(2):208-213. https://doi. org/10.1016/j.jfoodeng.2011.05.034

9. CAO, N.; FU, Y.; HE, J. 2007. Preparation and physical properties of soy protein isolate and gelatin composite films. Food Hydrocolloids. 21(7):1153-1162. https://doi. org/10.1016/j.foodhyd.2006.09.001

10. CAZÓN, P.; VELAZQUEZ, G.; RAMÍREZ, J.A.; VÁZQUEZ, M. 2017. Polysaccharide-based films and coatings for food packaging: A review. Food Hydrocolloids. 68:136-148. https://doi.org/10.1016/j.foodhyd.2016.09.009

11. CERQUEIRA, M.A.; SOUZA, B.W.S.; TEIXEIRA, J.A.; VICENTE, A. 2012. Effect of glycerol and corn oil on physicochemical properties of polysaccharide films - A comparative study. Food Hydrocolloids. 27(1):175-184. https://doi.org/10.1016/j.foodhyd.2011.07.007

12. CHAMBI, H.; GROSSO, C. 2006. Edible films produced with gelatin and casein cross-linked with transglutaminase. Food Research Internal. (Netherlands). 39(4):458-466. https:// doi.org/10.1016/j.foodres.2005.09.009

13. DE KRUIF, C.G.; WEINBRECK, F.; DE VRIES, R. 2004. Complex coacervation of proteins and anionic polysaccharides. Current Opinion in Colloid \& Interface Science. 9(5):340-349. https://doi.org/10.1016/j. cocis.2004.09.006

14. ESTEGHLAL, S.; NIAKOUSARI, M.; HOSSEINI, S.M.H. 2018. Physical and mechanical properties of gelatinCMC composite films under the influence of electrostatic interactions. Internal J. Biological Macromolecules. (Netherlands). 114:1-9. https://doi.org/10.1016/j. ijbiomac.2018.03.079

15. GALUS, S.; LENART, A. 2013. Development and characterization of composite edible films based on sodium alginate and pectin. J. Food Engineering. 15(4):459-465. https://doi.org/10.1016/j.jfoodeng.2012.03.006

16. GARAVAND, F.; ROUHI, M.; RAZAVI, S.H.; CACCIOTTI, I.; MOHAMMADI, R. 2017. Improving the integrity of natural biopolymer films used in food packaging by crosslinking approach: A review. Internal J. Biological 
Macromolecules. 104:687-707. https://doi.org/10.1016/j. ijbiomac.2017.06.093

17. GENNADIOS, A.; WELLER, C.L.; GOODING, C.H. 1994. Measurement errors in water vapor permeability of highly permeable, hydrophilic edible films. J. Food Engineering. 21(4):395-409. https://doi.org/10.1016/02608774(94)90062-0

18. GHASEMLOU, M.; KHODAIYAN, F.; OROMIEHIE, A. 2011. Physical, mechanical, barrier, and thermal properties of polyol-plasticized biodegradable edible film made from kefiran. Carbohydrate Polymers. (United Kingdom). 84(1):477-483. https://doi.org/10.1016/j. carbpol.2010.12.010

19. HAN, J.H.; FLOROS, J.D. 1997. Casting Antimicrobial Packaging Films and Measuring Their Physical Properties and Antimicrobial Activity. J. Plastic Film \& Sheeting. (United States). 13(4):287-298. https://doi. org $/ 10.1177 / 875608799701300405$

20. HASSAN, B.; CHATHA, S.A.S.; HUSSAIN, A.I.; ZIA, K.M.; AKHTAR, N. 2018. Recent advances on polysaccharides, lipids and protein based edible films and coatings: A review. Internal J. Biological Macromolecules. 109:1095-1107. https://doi.org/10.1016/j.ijbiomac.2017.11.097

21. JIMÉNEZ, A.; FABRA, M.J.; TALENS, P.; CHIRALT, A. 2012. Effect of re-crystallization on tensile, optical and water vapor barrier properties of corn starch films containing fatty acids. Food Hydrocolloids. 26(1):302-310. https:// doi.org/10.1016/j.foodhyd.2011.06.009

22. JRIDI, M.; HAJJI, S.; AYED, H.; LASSOUED, I.; MBAREK, A.; KAMMOUN, M.; SOUISSI, N.; NASRI, M. 2014. Physical, structural, antioxidant and antimicrobial properties of gelatin-chitosan composite edible films. Internal J. Biological Macromolecules. 67:373-379. https:/ / doi.org/10.1016/j.ijbiomac.2014.03.054

23. KHODAEI, D.; OLTROGGE, K.; HAMIDI-ESFAHANI, Z. 2019. Preparation and characterization of blended edible films manufactured using gelatin, tragacanth gum and, Persian gum. LWT. 117(2020):108617. https://doi. $\operatorname{org} / 10.1016 /$ j.lwt.2019.108617

24. LEE, K.Y.; SHIM, J.; LEE, H.G. 2004. Mechanical properties of gellan and gelatin composite films. Carbohydrate Polymers. 56(2):251-254. https://doi.org/10.1016/j. carbpol.2003.04.001

25. MANRICH, A.; MOREIRA, F.K.V.; OTONI, C.G.; LOREVICE, M.V.; MARTINS, M.A.; MATTTOSO, L.H.C.
2017. Hydrophobic edible films made up of tomato cutin and pectin. Carbohydrate Polymers. 164:83-91. https:// doi.org/10.1016/j.carbpol.2017.01.075

26. McCLEMENTS, D.J. 2005. Colloidal Interactions. Chapter 3. In: Food Emulsions: Principles, Practices, and Techniques. $3^{\text {rd }}$ Edition. London. CRC Press. p.39-82.

27. MONEDERO, F.M.; FABRA, M.J.; TALENS, P.; CHIRALT, A. 2010. Effect of calcium and sodium caseinates on physical characteristics of soy protein isolate-lipid films. J. Food Engineering. 97(2):228-234. https://doi.org/10.1016/j. jfoodeng.2009.10.014

28. PAN, H.; JIANG, B.; CHEN, J.; JIN, Z. 2014. Blendmodification of soy protein/lauric acid edible films using polysaccharides. Food Chemistry. (Netherlands). 151:1-6. https://doi.org/10.1016/j.foodchem.2013.11.075

29. PEREDA, M.; PONCE, A.G.; MARCOVICH, N.E.; RUSECKAITE, R.A.; MARTUCCI, J.F. 2011. Chitosangelatin composites and bi-layer films with potential antimicrobial activity. Food Hydrocolloids. 25(5):13721381. https://doi.org/10.1016/j.foodhyd.2011.01.001

30. QI, G.; LI, N.; SUN, X.S.; SHI, Y.; WANG, D. 2016. Effects of glycerol and nanoclay on physiochemical properties of camelina gum-based films. Carbohydrate Polymers. 152:747754. https://doi.org/10.1016/j.carbpol.2016.07.068

31. RHIM, J.W.; SHELLHAMMER, T.H. 2005. Lipid-based edible films and coatings. In: Innovations in Food Packaging. p. 362-383. https://doi.org/10.1016/B978-012311632$1 / 50053-\mathrm{X}$

32. SANYANG, M.L.; SAPUAN, S.M.; JAWAID, M.; ISHAK, M.R.; SAHARI, J. 2016. Effect of plasticizer type and concentration on physical properties of biodegradable films based on sugar palm (Arenga pinnata) starch for food packaging. J. Food Science and Technology. (United Kingdom). 53(1):326-336. https://doi.org/10.1007/ s13197-015-2009-7

33. TONGNUANCHAN, P.; BENJAKUL, S.; PRODPRAN, T. 2013. Physico-chemical properties, morphology and antioxidant activity of film from fish skin gelatin incorporated with root essential oils. J. Food Engineering.117(3):350-360. https://doi.org/10.1016/j.jfoodeng.2013.03.005

34. YILDIRIM, M.; MAHMUT, S.; SADIKOGL, H. 2019. Development and characterization of edible films based on modified corn starch and grape juice. Food Chemistry. 292:613. https://doi.org/10.1016/j.foodchem.2019.04.006 